\title{
Introducing the CME/MOC Corner
}

On behalf of the Liver Transplantation editorial team, we are very pleased to introduce the new "Liver Transplantation Continuing Medical Education (CME)/Maintenance of Certification (MOC) Corner." Later in the year, our editorial team will begin identifying 1 or 2 Original Articles/Review Articles to be featured in the CME/MOC Corner. These articles will be the sources for a test with short questions followed by a brief posttest evaluation. Credit for each test may be earned for up to 1 year after publication.

The American Association for the Study of Liver Diseases (AASLD) is accredited by the Accreditation Council for Continuing Medical Education to provide continuing medical education for physicians. The AASLD designates this journal-based CME activity for AMA PRA Category 1 Credit $^{\mathrm{TM}}$.

Successful completion of this CME activity, which includes participation in the evaluation component, enables the participant to earn up to $1 \mathrm{MOC}$ point in the American Board of Internal Medicine's (ABIM) MOC program. Participants will earn MOC points equivalent to the amount of $\mathrm{CME}$ credits claimed for the activity.

We hope that our readers find this activity useful and that it enhances the value of our journal in their professional lives.

\author{
Pratima Sharma, M.D., M.S. ${ }^{1}$ \\ Paul Martin, M.D. ${ }^{2}$ \\ Editor in-Chief, Liver Transplantation \\ ${ }^{1}$ Division of Gastroenterology and Hepatology \\ Michigan Medicine \\ University of Michigan \\ Ann Arbor, MI \\ ${ }^{2}$ University of Miami Health System \\ Miami, FL
}

Copyright (C) 2020 by the American Association for the Study of Liver

Diseases.

View this article online at wileyonlinelibrary.com.

DOI 10.1002/lt.25699 